CRYSTALLOGRAPHIC COMMUNICATIONS

ISSN 2056-9890

Received 11 September 2018

Accepted 25 September 2018

Edited by C. Rizzoli, Universita degli Studi di Parma, Italy

Keywords: crystal structure; thiazole derivative; hydrogen bonds; Hirshfeld surface analysis.

CCDC reference: 1869597

Supporting information: this article has supporting information at journals.iucr.org/e

\section{Crystal structure and Hirshfeld surface analysis of (Z)-4-chloro-N'-(4-oxothiazolidin-2-ylidene)- benzenesulfonohydrazide monohydrate}

\author{
Nikhila Pai, ${ }^{a}$ Sabine Foro ${ }^{\mathrm{b}}$ and B. Thimme Gowda ${ }^{\mathrm{a}, \mathrm{c} *}$
}

${ }^{\mathbf{a}}$ Department of Chemistry, Mangalore University, Mangalagangotri 574 199, Mangalore, India, ${ }^{\mathbf{b}}$ Institute of Materials Science, Darmstadt University of Technology, Alarich-Weiss-Str. 2, D-64287, Darmstadt, Germany, and ${ }^{\mathrm{c}}$ Karnataka State Rural Development and Panchayat Raj University, Gadag 582 101, India. *Correspondence e-mail: gowdabt@yahoo.com

The asymmetric unit of the title thiazole derivative containing a sulfonylhydrazinic moiety, $\mathrm{C}_{9} \mathrm{H}_{8} \mathrm{ClN}_{3} \mathrm{O}_{3} \mathrm{~S}_{2} \cdot \mathrm{H}_{2} \mathrm{O}$, consists of two independent molecules and two water molecules. The central parts of the molecules are twisted as both the molecules are bent at both the $\mathrm{S}$ and $\mathrm{N}$ atoms. In the crystal, $\mathrm{N}-\mathrm{H} \cdots \mathrm{N}, \mathrm{N}-$ $\mathrm{H} \cdots \mathrm{O}, \mathrm{C}-\mathrm{H} \cdots \mathrm{O}$ and $\mathrm{O}-\mathrm{H} \cdots \mathrm{O}$ hydrogen-bonding interactions connect the molecules, forming layers parallel to the $a b$ plane. Two-dimensional fingerprint plots associated with the Hirshfeld surface show that the largest contributions to the crystal packing come from $\mathrm{O} \cdots \mathrm{H} / \mathrm{H} \cdots \mathrm{O}(32.9 \%)$ and $\mathrm{H} \cdots \mathrm{H}(22.6 \%)$ interactions.

\section{Chemical context}

Sulfonamides are of interest as this class of compounds exhibits a wide array of biological activities such as antitumor, antibacterial, diuretic and hypoglycaemic activities (Kamal et $a l ., 2007)$. It has been reported that incorporation of hydrazine moieties increases the carbonic anhydrase inhibition activity (Winum et al., 2005). Along with the sulfonamide group, the presence of the 2-hydrazino-thiazole moiety enhances the pharmacological activities. The thiozoyl group is of interest because of its medicinal use in antitumor (Holla et al., 2003; Kappe et al., 2004), hyposensitive (Dash et al., 1980), anti-HIV (Patt et al., 1992), antimicrobial and anticancer agents (Frère et al., 2003). Sulfonylhydrazines and their derivatives can easily be prepared and are stable. We report herein the synthesis and structure of the title compound, which is a new thiazole compound containing a sulfonylhydrazinic moiety.<smiles>O=C1CS/C(=N/NS(=O)(=O)c2ccc(Cl)cc2)N1</smiles>

\section{Structural commentary}

The asymmetric unit of the title compound contains two independent molecules and two water molecules (Fig. 1). The $\mathrm{C} 8-\mathrm{O} 3$ and $\mathrm{C} 17-\mathrm{O} 6$ bond lengths of $1.202(5) \AA$, 1.218 (6) $\AA$, respectively, are consistent with $\mathrm{C}=\mathrm{O}$ doublebond character. Similarly, the values of the C7-N2 and C16N5 bond lengths [1.285 (5) and 1.276 (5) $\AA$, respectively] are 


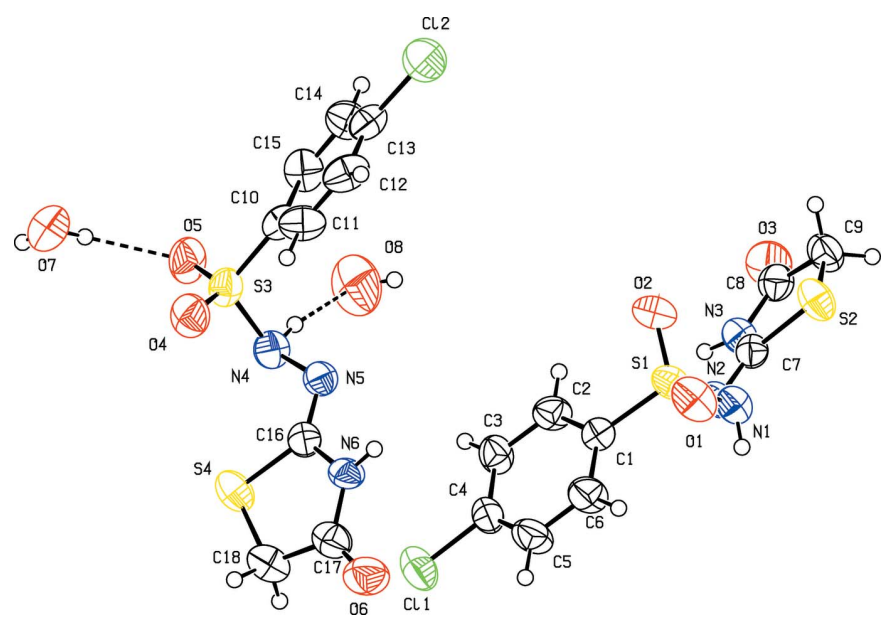

Figure 1

The molecular structure of the title compound showing displacement ellipsoids at the $50 \%$ probability level.

close to that of a typical $\mathrm{C}=\mathrm{N}$ double bond, while the longer $\mathrm{C} 7-\mathrm{N} 3$ and $\mathrm{C} 16-\mathrm{N} 6$ bond lengths of 1.370 (5) and 1.381 (5) $\AA$, respectively, are consistent with the normal C-N single bonds, indicating that the compound exists in the Schiff base form. Further, the N1-N2 and N4-N5 bond lengths of 1.440 (5) and 1.442 (5) $\AA$, respectively, and the S1-N1 and S3-N4 bond lengths of 1.644 (4) and 1.649 (4) A, respectively, are in agreement with single-bond character.

The central parts of both molecules are twisted as they are bent at the $\mathrm{S}$ (S1 and $\mathrm{S} 3$ ) and $\mathrm{N}(\mathrm{N} 2$ and $\mathrm{N} 5)$ atoms as indicated by the $\mathrm{C} 1-\mathrm{S} 1-\mathrm{N} 1-\mathrm{N} 2$ and $\mathrm{S} 1-\mathrm{N} 1-\mathrm{N} 2-\mathrm{C} 7$ torsion angles of $57.0(3)$ and $111.8(3)^{\circ}$, respectively, and by the $\mathrm{C} 10-\mathrm{S} 3-\mathrm{N} 4-\mathrm{N} 5$ and $\mathrm{S} 3-\mathrm{N} 4-\mathrm{N} 5-\mathrm{C} 16$ torsion angles of $57.6(3)$ and $109.7(3)^{\circ}$, respectively. The sulfonylhydrazide bond exists in the synclinal conformation preferred by aromatic sulfonamides (Purandara et al., 2017), with $\mathrm{C}-\mathrm{S}-$ $\mathrm{N}-\mathrm{N}$ torsion angles of $57.0(3)$ and $57.6(3)^{\circ}$ in the two independent molecules. The geometrical parameters for the thiazole and benzene rings are within the expected ranges and comparable with those of other substituted thiazoles or benzenesulfonylhydrazide derivatives (Zaharia et al., 2010). The $\mathrm{C} 7-\mathrm{S} 2-\mathrm{C} 9$ and $\mathrm{C} 16-\mathrm{S} 4-\mathrm{C} 18$ angles in the two molecules have the same value of $91.4(2)^{\circ}$, and it is similar to the angles typically observed in thiazole derivatives (Form et al., 1974). The thiazole rings are approximately planar (r.m.s. deviations of 0.011 and $0.029 \AA$ for S2/N3/C7-C9 and S4/N6/ C16-C18, respectively), and form dihedral angles of 26.18 (15) and $37.19(12)^{\circ}$ with the aromatic ring of the $p$-chlorophenylsulfonyl groups.

\section{Supramolecular features}

In the crystal, the two independent molecules are linked into dimers by pairs of $\mathrm{N}-\mathrm{H} \cdots \mathrm{N}$ hydrogen bonds, forming rings with an $R_{2}^{2}(8)$ graph-set motif. These dimers are connected by $\mathrm{C}-\mathrm{H} \cdots \mathrm{O}$ hydrogen bonds involving the thiazole $\mathrm{C}-\mathrm{H}$ and $\mathrm{a}$ sulfonyl $\mathrm{O}$ atom into chains running parallel to the $a$ axis
Table 1

Hydrogen-bond geometry $\left(\AA,^{\circ}\right)$.

\begin{tabular}{lllll}
\hline$D-\mathrm{H} \cdots A$ & $D-\mathrm{H}$ & $\mathrm{H} \cdots A$ & $D \cdots A$ & $D-\mathrm{H} \cdots A$ \\
\hline $\mathrm{N} 1-\mathrm{H} 1 N \cdots \mathrm{O} 7^{\text {i }}$ & $0.84(2)$ & $2.07(2)$ & $2.900(6)$ & $168(4)$ \\
$\mathrm{N} 3-\mathrm{H} 3 N \cdots \mathrm{N}^{\text {ii }}$ & $0.85(2)$ & $2.07(2)$ & $2.895(5)$ & $162(4)$ \\
$\mathrm{C} 9-\mathrm{H} 9 B \cdots \mathrm{O} 2^{\text {ii }}$ & 0.97 & 2.42 & $3.236(6)$ & 141 \\
$\mathrm{~N} 4-\mathrm{H} 4 N \cdots \mathrm{O} 8$ & $0.85(2)$ & $1.95(2)$ & $2.788(6)$ & $168(4)$ \\
$\mathrm{N} 6-\mathrm{H} 6 N \cdots \mathrm{N} 2^{\text {iii }}$ & $0.85(2)$ & $1.97(2)$ & $2.808(5)$ & $170(4)$ \\
$\mathrm{C} 15-\mathrm{H} 15 \cdots \mathrm{O} 1^{\text {iv }}$ & 0.93 & 2.55 & $3.355(5)$ & 145 \\
$\mathrm{O} 7-\mathrm{H} 71 \cdots \mathrm{O} 5$ & $0.82(2)$ & $2.08(3)$ & $2.868(5)$ & $162(6)$ \\
$\mathrm{O} 7-\mathrm{H} 72 \cdots 6^{\text {iv }}$ & $0.82(2)$ & $1.99(2)$ & $2.810(5)$ & $174(6)$ \\
$\mathrm{O} 8-\mathrm{H} 81 \cdots \mathrm{O} 4^{\text {ii }}$ & $0.82(2)$ & $2.35(6)$ & $2.987(6)$ & $136(7)$ \\
$\mathrm{O} 8-\mathrm{H} 81 \cdots 7^{\text {ii }}$ & $0.82(2)$ & $2.50(7)$ & $3.034(7)$ & $124(7)$ \\
$\mathrm{O} 8-\mathrm{H} 82 \cdots 3^{\text {iii }}$ & $0.83(2)$ & $2.37(3)$ & $3.159(7)$ & $159(8)$ \\
\hline
\end{tabular}

Symmetry codes: (i) $x-1, y+1, z$; (ii) $x-1, y, z$; (iii) $x+1, y, z$; (iv) $x, y-1, z$.

(Table 1, Fig. 2). The water molecules are involved both in the enforcement of the dimers through $\mathrm{N}-\mathrm{H} \cdots \mathrm{O}$ and $\mathrm{O}-\mathrm{H} \cdots \mathrm{O}$ hydrogen bonds, forming $R_{3}^{3}(9)$ rings, and in inter-chain $\mathrm{O}-$ $\mathrm{H}$... O hydrogen-bonding interactions, forming layers parallel to the $a b$ plane.

\section{Database survey}

Although a search in the Cambridge. Structural Database (CSD, Version 5.39, update of August 2018; Groom et al., 2016) revealed several reports of the crystal structure of sulfonamides and thiazole (Gowda et al., 2008, 2009), there are only a few reports on the crystal structures of sulfonylhydrazides functionalized by thiazole groups (Zaharia et al., 2010). Comparison of the structure of the title compound with that of $N^{\prime}$-(5-acetyl-4-methyl-4,5-dihydrothiazol-2-yl)benzenesulfonohydrazide (Zaharia et al., 2010) indicates that the electron-withdrawing chloro group does not impart sufficient inductive effect to reduce the electron density on the benzene ring, and that the ability of the aromatic $\mathrm{C}-\mathrm{H}$ groups to participate in $\mathrm{C}-\mathrm{H} \cdots \mathrm{O}$ interactions is very much reduced.

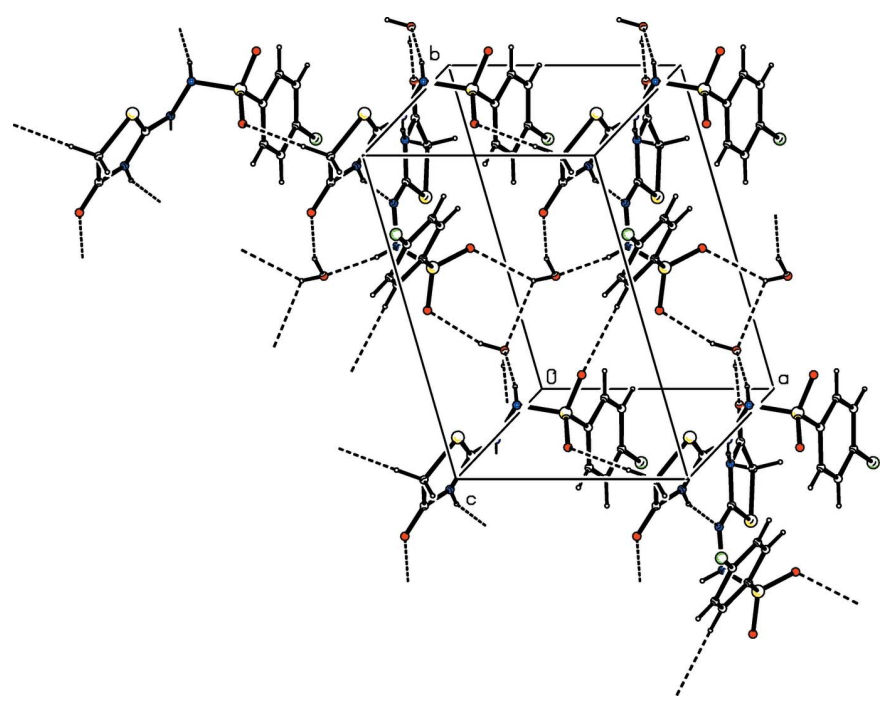

Figure 2

The molecular packing of the title compound, with hydrogen bonds (Table 1) shown as dashed lines. 
Partial double-bond character is observed between the hydrazinyl $\mathrm{N}$ atom and the adjacent benzothiazole moiety in 2-[2-(3-nitrobenzenesulfonyl)hydrazinyl]-1,3-benzothiazole (Morscher et al., 2018). The orientation of the thiazole ring in the title compound is similar to that of $(Z)$-methyl 2-[(Z)-4oxo-2-(2-tosylhydrazono)thiazolidin-5-ylidene]acetate and (Z)-methyl-2-[(Z)-2-(ethylimino)-4-oxo-3-(phenylamino)thiazolidin-5-ylidene]acetate (Hassan et al., 2016). The molecule of $\quad N^{\prime}$-\{3-[3-(trifluoromethyl)phenyl]-1,3-thiazol-2(3H)-ylidene \}benzenesulfonohydrazide (Chen et al., 2015) is observed to have a Schiff base conformation.

\section{Hirshfeld Surface Analysis}

In order to explore the role of weak intermolecular interactions in the crystal packing, Hirshfeld surfaces $\left(d_{\text {norm }}\right)$ and related fingerprint plots were generated using CrystalExplorer17.5 (McKinnon et al., 2007; Spackman et al., 2008; Spackman \& Jayatilaka, 2009; Wolff et al., 2012). The threedimensional molecular Hirshfeld surfaces were generated using a high standard surface resolution over a colour scale of -0.6355 to 1.5137 a.u. for $d_{\text {norm }}$. To identify the normalized contacts, the $d_{\text {norm }}$ function is used, which is expressed as; $d_{\text {norm }}=\left(d_{\mathrm{i}}-r_{\mathrm{i}}^{\mathrm{vdw}}\right) / r_{\mathrm{i}}{ }^{\mathrm{vdw}}+\left(d_{\mathrm{e}}-r_{\mathrm{e}}^{\mathrm{vdw}}\right) / r_{\mathrm{e}}{ }^{\mathrm{vdw}}$ (Shit et al., 2016), where $d_{\mathrm{i}}$ and $d_{\mathrm{e}}$ are the distances from internal and external atoms to the Hirshfeld surface and $r_{\mathrm{i}}^{\mathrm{vdw}}$ and $r_{\mathrm{e}}{ }^{\mathrm{vdw}}$ are the van der Waals radii of the atoms inside and outside the surface. On the Hirshfeld surfaces mapped over $d_{\text {norm }}$ (Fig. 3), strong $\mathrm{N}-$ $\mathrm{H} \cdots \mathrm{N}$ and $\mathrm{S}-\mathrm{O} \cdots \mathrm{H}$ interactions are observed as red spots close to atoms N5, N6 and O6. Furthermore, the two-dimensional fingerprint plots indicate that the largest contributions are from $\mathrm{O} \cdots \mathrm{H} / \mathrm{H} \cdots \mathrm{O}$ contacts, which contribute $32.9 \%$ to the Hirshfeld surface (Fig. $4 a$ ) with $d_{\mathrm{i}}+d_{\mathrm{e}} \sim 1.9 \AA$. The

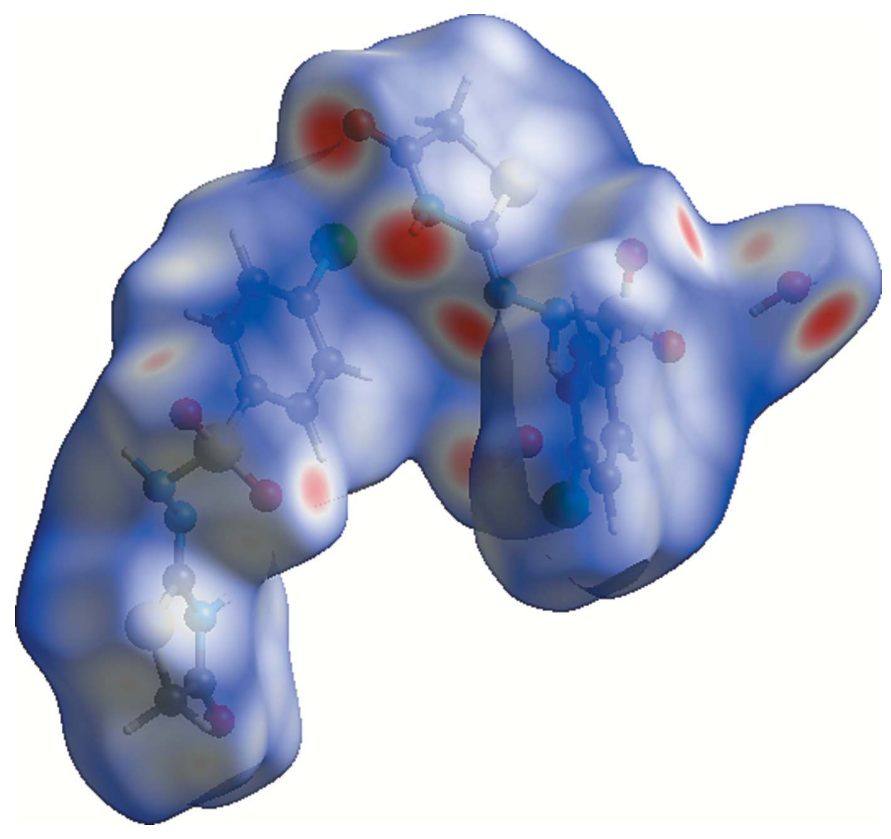

Figure 3

View of the Hirshfeld surface mapped over $d_{\text {norm }}$.

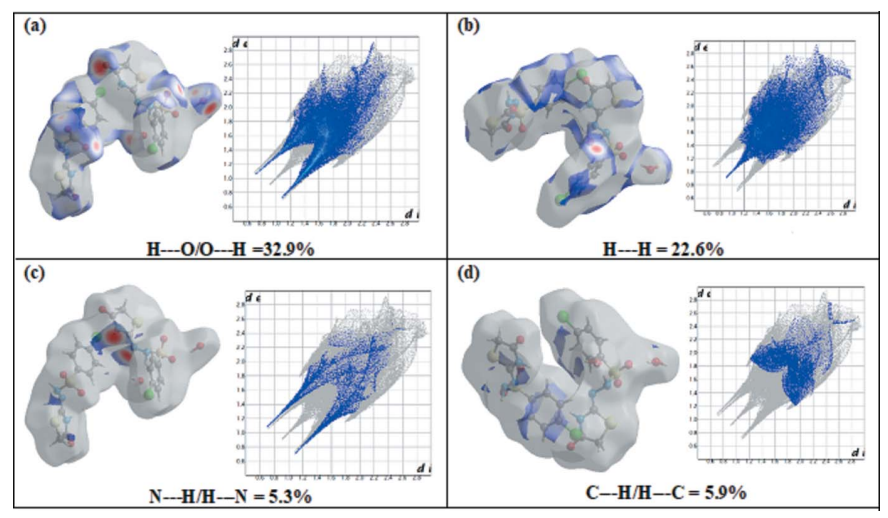

Figure 4

The two dimensional fingerprint (FP) plot for the title compound, delineated into $(a) \mathrm{O} \cdots \mathrm{H} / \mathrm{H} \cdots \mathrm{O},(b) \mathrm{H} \cdots \mathrm{H},(c) \mathrm{N} \cdots \mathrm{H} / \mathrm{H} \cdots \mathrm{N}$ and $(d)$ $\mathrm{C} \cdots \mathrm{H} / \mathrm{H} \cdots \mathrm{C}$ interactions. $d_{\text {norm }}$ surfaces for each plot indicating the relevant surface patches associated with the specific contacts are shown on the left.

presence of water molecules in the unit cell provides the largest contribution to the stability of the crystal packing. The next largest contributor is from $\mathrm{H} \cdots \mathrm{H}$ interactions, which contribute $22.6 \%$. A single sharp spike can be seen in the middle region of the plot, at $d_{\mathrm{i}}=d_{\mathrm{e}}=0.9 \AA$ (Fig. $4 b$ ). The $\mathrm{N} \cdots \mathrm{H}$ contacts, which refer to $\mathrm{N}-\mathrm{H} \cdots \mathrm{N}$ interactions, contribute $5.3 \%$ to the surface. Two sharp spikes having $d_{\mathrm{i}}+d_{\mathrm{e}}$ $=1.8 \AA$ (Fig. $4 c$ ) are observed. The $\mathrm{C} \cdot \cdots \mathrm{H}$ contacts contribute $5.9 \%$ to the Hirshfeld surface, featuring a wide region with $d_{\mathrm{i}}+$ $d_{\mathrm{e}}=3.1 \AA$ (Fig. $4 d$ ). The different interatomic contacts and percentage contributions to the Hirshfeld surface are $\mathrm{Cl} \cdots \mathrm{H} /$ $\mathrm{H} \cdots \mathrm{Cl}(8.3 \%), \mathrm{S} \cdots \mathrm{H} / \mathrm{H} \cdots \mathrm{S}(6.1 \%), \mathrm{Cl} \cdots \mathrm{O} / \mathrm{O} \cdots \mathrm{Cl}(3.0 \%)$, $\mathrm{Cl} \cdots \mathrm{C} / \mathrm{C} \cdots \mathrm{Cl}(2.4 \%), \mathrm{S} \cdots \mathrm{O} / \mathrm{O} \cdots \mathrm{S}(1.7 \%)$, and $\mathrm{C} \cdots \mathrm{O} / \mathrm{O} \cdots \mathrm{C}$ $(1.6 \%)$ as depicted in the fingerprint plots (Fig. $5 a-f$ ).
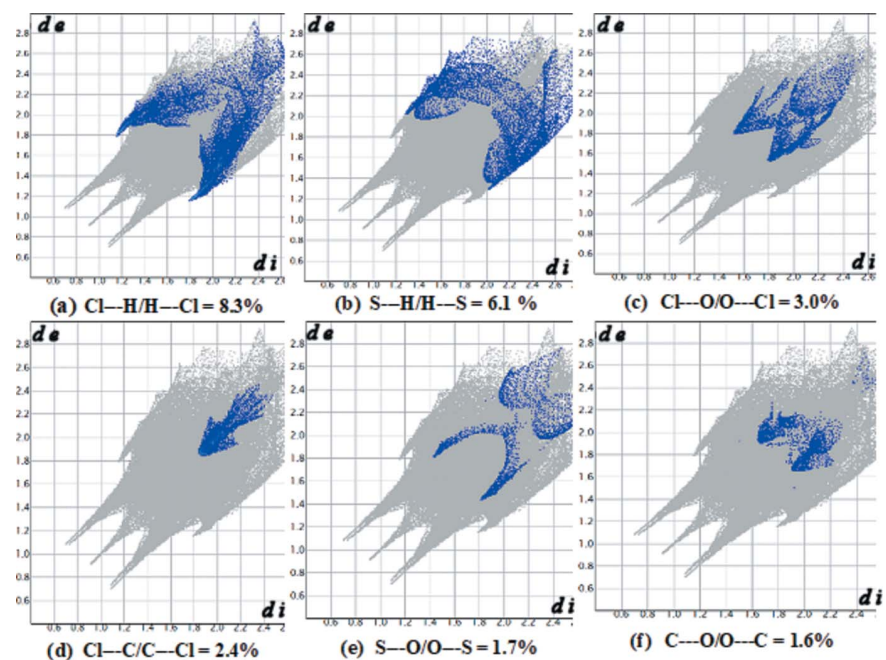

(b) $\mathrm{S}-\mathrm{H} / \mathrm{H}-\mathrm{S}=6.1 \%$

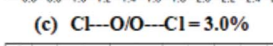

(d) $\mathrm{Cl}-\mathrm{C} / \mathrm{C}-\mathrm{-Cl}=2.4 \%$

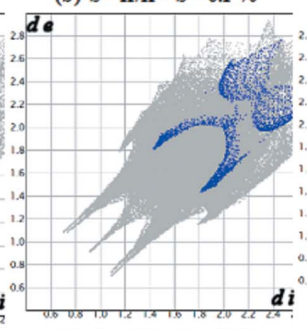

(e) $\mathrm{S}-\mathrm{-O} / \mathrm{O}-\mathrm{-S}=1.7 \%$

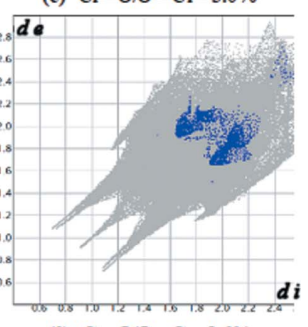

(f) $\mathrm{C}-\mathrm{-O} / \mathrm{O}-\mathrm{C}=1.6 \%$

Figure 5

Fingerprint plots of interactions, listing their percentage contributions: (a) $\mathrm{Cl} \cdots \mathrm{H} / \mathrm{H} \cdots \mathrm{Cl}$, (b) $\mathrm{S} \cdots \mathrm{H} / \mathrm{H} \cdots \mathrm{S}$, (c) $\mathrm{Cl} \cdots \mathrm{O} / \mathrm{O} \cdots \mathrm{Cl}$, (d) $\mathrm{Cl} \cdots \mathrm{Cl}$ $\mathrm{C} \cdots \mathrm{Cl},(e) \mathrm{S} \cdots \mathrm{O} / \mathrm{O} \cdots \mathrm{S}$ and $(f) \mathrm{C} \cdots \mathrm{O} / \mathrm{O} \cdots \mathrm{C}$. 


\section{Synthesis and crystallization}

4-Chloro- $N^{\prime}$-(4-oxo-4,5-dihydro-1,3-thiazol-2-yl)benzene-1sulfonohydrazide was prepared by adding 4-chloro benzenesulfonyl chloride $(0.02 \mathrm{~mol})$ under stirring to a solution of thiosemicarbazide $(0.02 \mathrm{~mol})$ in $5 \%$ aqueous $\mathrm{NaOH}$ solution $(20 \mathrm{ml})$. The reaction mixture was stirred at room temperature for $1 \mathrm{~h}$, then diluted twofold with water and neutralized with glacial acetic acid. The solid 2-(4-chlorobenzene-1-sulfonyl)hydrazine-1-carbothioamide $(A)$ obtained was crystallized from acetic acid. Monochloroacetic acid (0.01 mol) and anhydrous sodium acetate $(0.04 \mathrm{~mol})$ were added to $A$ $(0.01 \mathrm{~mol})$ in glacial acetic acid. The reaction mixture was refluxed for $8-10 \mathrm{~h}$ and the completion of the reaction was checked by TLC. The reaction mixture was then poured into cold water. The resulted precipitate of the title compound was separated by vacuum filtration. Prismatic colourless single crystals of the title compound were grown from a mixture of acetonitrile-DMF $(5: 1 v / v)$ by slow evaporation of the solvent. The purity of the compound was checked by TLC and characterized by IR spectroscopy. The characteristic IR absorptions observed at 3095.9, 1639.5, 1458.7, 1343.2, 1139.4, and $1215.7 \mathrm{~cm}^{-1}$ correspond to $\mathrm{N}-\mathrm{H}, \mathrm{C}=\mathrm{O}, \mathrm{C}=\mathrm{N}, \mathrm{S}=\mathrm{O}$ asymmetric and symmetric, and $\mathrm{C}-\mathrm{S}$ absorptions, respectively. The ${ }^{1} \mathrm{H}$ and ${ }^{13} \mathrm{C}$ spectra of the title compound are as follows: ${ }^{1} \mathrm{H}\left(400 \mathrm{MHz}, \mathrm{DMSO}-d_{6}\right) ; \delta 3.45\left(d, 2 \mathrm{H},-\mathrm{CH}_{2}\right), 7.68-7.86(m$, $4 \mathrm{H}, \mathrm{Ar}-\mathrm{H}), 10.01(s, 1 \mathrm{H}), 11.96(s, 1 \mathrm{H}) .{ }^{13} \mathrm{C} \mathrm{NMR}(400 \mathrm{MHz}$, DMSO- $\left.d_{6}\right) ; \delta 36.8,128.4,129.1,131.1,132.5,133.9,137.2,165.4$, 185.5 .

\section{Refinement}

Crystal data, data collection and structure refinement details are summarized in Table 2. $\mathrm{H}$ atoms bonded to $\mathrm{C}$ were positioned with idealized geometry using a riding model with $\mathrm{C}-$ $\mathrm{H}=0.93 \AA$ (aromatic) or $0.97 \AA$ (methylene). The $\mathrm{H}$ atoms of the $\mathrm{NH}$ groups and the $\mathrm{H}$ atoms of the water molecules were located in a difference-Fourier map and later refined with the $\mathrm{N}-\mathrm{H}$ and $\mathrm{O}-\mathrm{H}$ bond lengths constrained to be 0.86 (2) and 0.82 (2) $\AA$, respectively. All $\mathrm{H}$ atoms were refined with isotropic displacement parameters set at $1.2 U_{\mathrm{eq}}$ of the parent atom.

\section{Acknowledgements}

The authors thank the SAIF, Panjab University, for use of the NMR facility.

\section{Funding information}

NP thanks the Department of Science and Technology, Government of India, New Delhi, for a research fellowship under its PURSE Program and BTG thanks the University Grants Commission, Government of India, New Delhi, for a special grant under a UGC-BSR one-time grant to faculty.
Table 2

Experimental details.

\begin{tabular}{|c|c|}
\hline \multicolumn{2}{|l|}{ Crystal data } \\
\hline Chemical formula & $\mathrm{C}_{9} \mathrm{H}_{8} \mathrm{ClN}_{3} \mathrm{O}_{3} \mathrm{~S}_{2} \cdot \mathrm{H}_{2} \mathrm{O}$ \\
\hline$M_{\mathrm{r}}$ & 323.77 \\
\hline Crystal system, space group & Triclinic, $P \overline{1}$ \\
\hline Temperature $(\mathrm{K})$ & 293 \\
\hline$a, b, c(\AA)$ & $7.6276(6), 11.090(1), 17.116(2)$ \\
\hline$\alpha, \beta, \gamma\left({ }^{\circ}\right)$ & $96.95(1), 99.49(1), 106.08$ (1) \\
\hline$V\left(\AA^{3}\right)$ & $1350.8(2)$ \\
\hline$Z$ & 4 \\
\hline Radiation type & Mo $K \alpha$ \\
\hline$\mu\left(\mathrm{mm}^{-1}\right)$ & 0.60 \\
\hline Crystal size (mm) & $0.42 \times 0.20 \times 0.06$ \\
\hline \multicolumn{2}{|l|}{ Data collection } \\
\hline Diffractometer & $\begin{array}{l}\text { Oxford Diffraction Xcalibur Single } \\
\text { Crystal X-ray diffractometer } \\
\text { with a Sapphire CCD detector }\end{array}$ \\
\hline Absorption correction & $\begin{array}{l}\text { Multi-scan (CrysAlis RED; Oxford } \\
\text { Diffraction, 2009) }\end{array}$ \\
\hline$T_{\min }, T_{\max }$ & $0.785,0.965$ \\
\hline $\begin{array}{l}\text { No. of measured, independent and } \\
\text { observed }[I>2 \sigma(I)] \text { reflections }\end{array}$ & $8631,4935,3375$ \\
\hline$R_{\text {int }}$ & 0.026 \\
\hline$(\sin \theta / \lambda)_{\max }\left(\AA^{-1}\right)$ & 0.602 \\
\hline \multicolumn{2}{|l|}{ Refinement } \\
\hline$R\left[F^{2}>2 \sigma\left(F^{2}\right)\right], w R\left(F^{2}\right), S$ & $0.058,0.161,1.05$ \\
\hline No. of reflections & 4935 \\
\hline No. of parameters & 367 \\
\hline No. of restraints & 8 \\
\hline $\mathrm{H}$-atom treatment & $\begin{array}{l}\mathrm{H} \text { atoms treated by a mixture of } \\
\text { independent and constrained } \\
\text { refinement }\end{array}$ \\
\hline$\Delta \rho_{\max }, \Delta \rho_{\min }\left(\mathrm{e} \AA^{-3}\right)$ & $0.52,-0.25$ \\
\hline
\end{tabular}

Computer programs: CrysAlis CCD and CrysAlis RED (Oxford Diffraction, 2009), SHELXS2013 (Sheldrick, 2008), SHELXL2014 (Sheldrick, 2015) and PLATON (Spek, 2009).

\section{References}

Chen, W., Wang, H.-A., Wei, W., Li, Y.-X., Hua, X.-W., Song, H.-B., Yu, S.-J. \& Li, Z.-M. (2015). Chin. J. Struct. Chem. 34, 503-509.

Dash, B., Patra, M. \& Praharaj, S. (1980). Indian J. Chem. 19B, 894897.

Form, G. R., Raper, E. S. \& Downie, T. C. (1974). Acta Cryst. B30, 342-348.

Frère, S., Thiéry, V., Bailly, C. \& Besson, T. (2003). Tetrahedron, 59, 773-779.

Gowda, B. T., Foro, S., Babitha, K. S. \& Fuess, H. (2008). Acta Cryst. E64, o2190.

Gowda, B. T., Foro, S., Nirmala, P. G. \& Fuess, H. (2009). Acta Cryst. E65, o2763.

Groom, C. R., Bruno, I. J., Lightfoot, M. P. \& Ward, S. C. (2016). Acta Cryst. B72, 171-179.

Hassan, A. A., Mohamed, N. K., El-Shaieb, K. M. A., Tawfeek, H. N., Bräse, S. \& Nieger, M. (2016). J. Heterocycl. Chem. 53, 46-50.

Holla, B. S., Malini, V. K., Rao, B. S., Sarojini, K. B. \& Kumari, N. S. (2003). Eur. J. Med. Chem. 38, 313-318.

Kamal, A., Khan, M. N. A., Srinivasa Reddy, K. \& Rohini, K. (2007). Bioorg. Med. Chem. 15, 1004-1013.

Kappe, O. C. (2004). Angew. Chem. Int. Ed. 43, 6250-6284.

McKinnon, J. J., Jayatilaka, D. \& Spackman, M. A. (2007). Chem. Commun. pp. 3814-3816.

Morscher, A., de Souza, M. V. N., Wardell, J. L. \& Harrison, W. T. A. (2018). Acta Cryst. E74, 673-677.

Oxford Diffraction (2009). CrysAlis CCD and CrysAlis RED. Oxford Diffraction Ltd., Abingdon, England. 
Patt, W. C., Hamilton, H. W., Taylor, M. D., Ryan, M. J., Taylor, D. G. Jr, Connolly, C. J. C., Doherty, A. M., Klutchko, S. R., Sircar, I., Steinbaugh, B. A., Batley, B. L., Painchaud, C. A., Rapundalo, S. T., Michniewicz, B. M. \& Oslon, S. C. (1992). J. Med. Chem. 35, 2562-2572.

Purandara, H., Foro, S. \& Thimme Gowda, B. (2017). Acta Cryst. E73, 1683-1686.

Sheldrick, G. M. (2008). Acta Cryst. A64, 112-122.

Sheldrick, G. M. (2015). Acta Cryst. C71, 3-8.

Shit, S., Marschner, C. \& Mitra, S. (2016). Acta Chim. Slovenica, 63, 129-137.

Spackman, M. A. \& Jayatilaka, D. (2009). CrystEngComm, 11, 19-32.
Spackman, M. A., McKinnon, J. J. \& Jayatilaka, D. (2008). CrystEngComm, 10, 377-388.

Spek, A. L. (2009). Acta Cryst. D65, 148-155.

Winum, J. Y., Dogné, J. M., Casini, A., de Leval, X., Montero, J. L., Scozzafava, A., Vullo, D., Innocenti, A. \& Supuran, C. T. (2005). J. Med. Chem. 48, 2121-2125.

Wolff, S. K., Grimwood, D. J., McKinnon, J. J., Turner, M. J., Jayatilaka, D. \& Spackman, M. A. (2012). Crystal Explorer3.1. University of Western Australia.

Zaharia, V., Curticapenan, M., Palibroda, N., Vlasa, M. \& Silvestru, A. (2010). Rev. Roum. Chim. 55, 831-841. 


\section{supporting information}

Acta Cryst. (2018). E74, 1569-1573 [https://doi.org/10.1107/S2056989018013658]

\section{Crystal structure and Hirshfeld surface analysis of (Z)-4-chloro-N'-(4-oxothia-} zolidin-2-ylidene)benzenesulfonohydrazide monohydrate

\section{Nikhila Pai, Sabine Foro and B. Thimme Gowda}

Computing details

Data collection: CrysAlis CCD (Oxford Diffraction, 2009); cell refinement: CrysAlis RED (Oxford Diffraction, 2009); data reduction: CrysAlis RED (Oxford Diffraction, 2009); program(s) used to solve structure: SHELXS2013 (Sheldrick, 2008); program(s) used to refine structure: SHELXL2014 (Sheldrick, 2015); molecular graphics: PLATON (Spek, 2009); software used to prepare material for publication: SHELXL2014 (Sheldrick, 2015).

(Z)-4-Chloro-N'-(4-oxothiazolidin-2-ylidene)benzenesulfonohydrazide monohydrate

\section{Crystal data}

\section{$\mathrm{C}_{9} \mathrm{H}_{8} \mathrm{ClN}_{3} \mathrm{O}_{3} \mathrm{~S}_{2} \cdot \mathrm{H}_{2} \mathrm{O}$}

$M_{r}=323.77$

Triclinic, $P \overline{1}$

$a=7.6276(6) \AA$

$b=11.090(1) \AA$

$c=17.116(2) \AA$

$\alpha=96.95(1)^{\circ}$

$\beta=99.49(1)^{\circ}$

$\gamma=106.08(1)^{\circ}$

$V=1350.8(2) \AA^{3}$

\section{Data collection}

Oxford Diffraction Xcalibur Single Crystal Xray diffractometer with a Sapphire CCD detector Radiation source: Enhance (Mo) X-ray Source Rotation method data acquisition using $\omega$ scans Absorption correction: multi-scan (CrysAlis RED; Oxford Diffraction, 2009)

$T_{\min }=0.785, T_{\max }=0.965$

\section{Refinement}

Refinement on $F^{2}$

Least-squares matrix: full

$R\left[F^{2}>2 \sigma\left(F^{2}\right)\right]=0.058$

$w R\left(F^{2}\right)=0.161$

$S=1.05$

4935 reflections

367 parameters

8 restraints

$$
\begin{aligned}
& Z=4 \\
& F(000)=664 \\
& D_{\mathrm{x}}=1.592 \mathrm{Mg} \mathrm{m}^{-3}
\end{aligned}
$$

Mo $K \alpha$ radiation, $\lambda=0.71073 \AA$

Cell parameters from 2682 reflections

$\theta=2.8-27.8^{\circ}$

$\mu=0.60 \mathrm{~mm}^{-1}$

$T=293 \mathrm{~K}$

Prism, colourless

$0.42 \times 0.20 \times 0.06 \mathrm{~mm}$

8631 measured reflections

4935 independent reflections

3375 reflections with $I>2 \sigma(I)$

$R_{\text {int }}=0.026$

$\theta_{\text {max }}=25.4^{\circ}, \theta_{\min }=2.8^{\circ}$

$h=-9 \rightarrow 5$

$k=-13 \rightarrow 13$

$l=-20 \rightarrow 20$

Hydrogen site location: mixed

$\mathrm{H}$ atoms treated by a mixture of independent and constrained refinement

$w=1 /\left[\sigma^{2}\left(F_{\mathrm{o}}^{2}\right)+(0.0767 P)^{2}+1.1929 P\right]$ where $P=\left(F_{\mathrm{o}}^{2}+2 F_{\mathrm{c}}^{2}\right) / 3$

$(\Delta / \sigma)_{\max }=0.001$

$\Delta \rho_{\max }=0.52$ e $\AA^{-3}$

$\Delta \rho_{\min }=-0.25$ e $\AA^{-3}$ 


\section{Special details}

Geometry. All esds (except the esd in the dihedral angle between two 1.s. planes) are estimated using the full covariance matrix. The cell esds are taken into account individually in the estimation of esds in distances, angles and torsion angles; correlations between esds in cell parameters are only used when they are defined by crystal symmetry. An approximate (isotropic) treatment of cell esds is used for estimating esds involving l.s. planes.

Fractional atomic coordinates and isotropic or equivalent isotropic displacement parameters $\left(\AA^{2}\right)$

\begin{tabular}{|c|c|c|c|c|}
\hline & $x$ & $y$ & $z$ & $U_{\text {iso }} * / U_{\text {eq }}$ \\
\hline $\mathrm{Cl1}$ & $0.33684(17)$ & $0.77231(13)$ & $0.00096(7)$ & $0.0689(4)$ \\
\hline S1 & $0.23228(14)$ & $1.01774(10)$ & $0.33163(6)$ & $0.0479(3)$ \\
\hline S2 & $-0.21540(16)$ & $0.96967(12)$ & $0.42899(7)$ & $0.0594(3)$ \\
\hline $\mathrm{O} 1$ & $0.3605(4)$ & $1.1427(3)$ & $0.35513(19)$ & $0.0659(9)$ \\
\hline $\mathrm{O} 2$ & $0.2255(4)$ & $0.9268(3)$ & $0.38508(18)$ & $0.0626(8)$ \\
\hline $\mathrm{O} 3$ & $-0.6004(5)$ & $0.6450(3)$ & $0.3600(2)$ & $0.0732(10)$ \\
\hline N1 & $0.0248(5)$ & $1.0382(3)$ & $0.3165(2)$ & $0.0484(8)$ \\
\hline $\mathrm{H} 1 \mathrm{~N}$ & $0.024(6)$ & $1.086(4)$ & $0.282(2)$ & $0.058^{*}$ \\
\hline N2 & $-0.1246(4)$ & $0.9225(3)$ & $0.2827(2)$ & $0.0471(8)$ \\
\hline N3 & $-0.3702(4)$ & $0.7765(3)$ & $0.3134(2)$ & $0.0460(8)$ \\
\hline $\mathrm{H} 3 \mathrm{~N}$ & $-0.380(6)$ & $0.721(3)$ & $0.2726(19)$ & $0.055^{*}$ \\
\hline $\mathrm{C} 1$ & $0.2676(5)$ & $0.9482(4)$ & $0.2392(2)$ & $0.0421(9)$ \\
\hline $\mathrm{C} 2$ & $0.2102(6)$ & $0.8168(4)$ & $0.2167(3)$ & $0.0515(10)$ \\
\hline $\mathrm{H} 2$ & 0.1557 & 0.7651 & 0.2505 & $0.062^{*}$ \\
\hline $\mathrm{C} 3$ & $0.2345(6)$ & $0.7627(4)$ & $0.1432(3)$ & $0.0523(11)$ \\
\hline $\mathrm{H} 3$ & 0.1967 & 0.6747 & 0.1273 & $0.063^{*}$ \\
\hline $\mathrm{C} 4$ & $0.3146(5)$ & $0.8409(4)$ & $0.0946(2)$ & $0.0475(10)$ \\
\hline $\mathrm{C} 5$ & $0.3755(7)$ & $0.9718(5)$ & $0.1168(3)$ & $0.0617(12)$ \\
\hline H5 & 0.4315 & 1.0231 & 0.0831 & $0.074 *$ \\
\hline C6 & $0.3514(7)$ & $1.0250(4)$ & $0.1899(3)$ & $0.0577(12)$ \\
\hline H6 & 0.3920 & 1.1131 & 0.2060 & $0.069^{*}$ \\
\hline $\mathrm{C} 7$ & $-0.2275(5)$ & $0.8889(4)$ & $0.3335(2)$ & $0.0419(9)$ \\
\hline $\mathrm{C} 8$ & $-0.4741(6)$ & $0.7422(5)$ & $0.3700(3)$ & $0.0522(11)$ \\
\hline C9 & $-0.4074(6)$ & $0.8429(5)$ & $0.4445(3)$ & $0.0600(12)$ \\
\hline H9A & -0.3669 & 0.8068 & 0.4905 & $0.072 *$ \\
\hline H9B & -0.5080 & 0.8761 & 0.4549 & $0.072^{*}$ \\
\hline $\mathrm{Cl} 2$ & $0.8418(2)$ & $0.63805(14)$ & $0.57661(8)$ & $0.0805(4)$ \\
\hline S3 & $0.78365(15)$ & $0.43436(10)$ & $0.21411(7)$ & $0.0511(3)$ \\
\hline S4 & $0.74715(17)$ & $0.59857(12)$ & $0.02143(7)$ & $0.0603(3)$ \\
\hline O4 & $0.9602(4)$ & $0.4892(3)$ & $0.1948(2)$ & $0.0670(9)$ \\
\hline O5 & $0.7003(5)$ & $0.2980(3)$ & $0.1978(2)$ & $0.0691(9)$ \\
\hline O6 & $0.8569(5)$ & 0.9655 (4) & $0.0519(2)$ & $0.0762(10)$ \\
\hline N4 & $0.6297(5)$ & $0.4853(3)$ & $0.1590(2)$ & $0.0501(9)$ \\
\hline $\mathrm{H} 4 \mathrm{~N}$ & $0.541(5)$ & $0.458(4)$ & $0.183(3)$ & $0.060^{*}$ \\
\hline N5 & $0.6762(5)$ & $0.6224(3)$ & $0.1738(2)$ & $0.0468(8)$ \\
\hline N6 & $0.7780(5)$ & $0.8026(3)$ & $0.12079(19)$ & $0.0473(8)$ \\
\hline $\mathrm{H} 6 \mathrm{~N}$ & $0.804(6)$ & $0.847(4)$ & $0.1678(15)$ & $0.057^{*}$ \\
\hline $\mathrm{C} 10$ & $0.8011(5)$ & $0.4900(4)$ & $0.3167(3)$ & $0.0461(10)$ \\
\hline C11 & $0.9365(6)$ & $0.6028(4)$ & $0.3554(3)$ & $0.0593(12)$ \\
\hline
\end{tabular}




$\begin{array}{lllll}\text { H11 } & 1.0191 & 0.6482 & 0.3273 & 0.071^{*} \\ \text { C12 } & 0.9484(6) & 0.6473(4) & 0.4349(3) & 0.0605(12) \\ \text { H12 } & 1.0383 & 0.7233 & 0.4607 & 0.073^{*} \\ \text { C13 } & 0.8276(6) & 0.5797(4) & 0.4762(3) & 0.0540(11) \\ \text { C14 } & 0.6935(6) & 0.4666(5) & 0.4392(3) & 0.0611(12) \\ \text { H14 } & 0.6137 & 0.4207 & 0.4682 & 0.073^{*} \\ \text { C15 } & 0.6792(6) & 0.4226(4) & 0.3595(3) & 0.0566(11) \\ \text { H15 } & 0.5875 & 0.3474 & 0.3339 & 0.068^{*} \\ \text { C16 } & 0.7281(5) & 0.6717(4) & 0.1146(2) & 0.0438(9) \\ \text { C17 } & 0.8171(6) & 0.8523(5) & 0.0550(3) & 0.0575(12) \\ \text { C18 } & 0.8040(7) & 0.7499(5) & -0.0134(3) & 0.0652(13) \\ \text { H18A } & 0.9219 & 0.7657 & -0.0308 & 0.078^{*} \\ \text { H18B } & 0.7080 & 0.7488 & -0.0586 & 0.078^{*} \\ \text { O7 } & 0.9751(5) & 0.1699(4) & 0.1821(3) & 0.0766(10) \\ \text { H71 } & 0.880(5) & 0.191(6) & 0.182(4) & 0.092^{*} \\ \text { H72 } & 0.933(8) & 0.112(4) & 0.143(3) & 0.092^{*} \\ \text { O8 } & 0.3062(6) & 0.4099(6) & 0.2195(4) & 0.1111(16) \\ \text { H81 } & 0.195(4) & 0.390(7) & 0.199(4) & 0.133^{*} \\ \text { H82 } & 0.303(12) & 0.472(5) & 0.250(4) & 0.133^{*}\end{array}$

Atomic displacement parameters $\left(\AA^{2}\right)$

\begin{tabular}{lllllll}
\hline & $U^{11}$ & $U^{22}$ & $U^{33}$ & $U^{12}$ & $U^{13}$ & $U^{23}$ \\
\hline C11 & $0.0697(8)$ & $0.0852(9)$ & $0.0517(7)$ & $0.0274(7)$ & $0.0175(6)$ & $-0.0042(6)$ \\
S1 & $0.0486(6)$ & $0.0477(6)$ & $0.0378(6)$ & $0.0020(5)$ & $0.0078(4)$ & $0.0023(5)$ \\
S2 & $0.0572(7)$ & $0.0670(8)$ & $0.0452(6)$ & $0.0120(6)$ & $0.0114(5)$ & $-0.0085(5)$ \\
O1 & $0.0601(19)$ & $0.057(2)$ & $0.059(2)$ & $-0.0078(15)$ & $0.0119(15)$ & $-0.0071(15)$ \\
O2 & $0.070(2)$ & $0.072(2)$ & $0.0449(18)$ & $0.0156(17)$ & $0.0139(15)$ & $0.0183(16)$ \\
O3 & $0.059(2)$ & $0.068(2)$ & $0.088(3)$ & $0.0044(18)$ & $0.0237(18)$ & $0.0185(19)$ \\
N1 & $0.049(2)$ & $0.039(2)$ & $0.049(2)$ & $0.0029(16)$ & $0.0122(17)$ & $-0.0008(15)$ \\
N2 & $0.0460(19)$ & $0.046(2)$ & $0.0420(19)$ & $0.0047(16)$ & $0.0111(16)$ & $-0.0001(15)$ \\
N3 & $0.0411(18)$ & $0.049(2)$ & $0.042(2)$ & $0.0083(16)$ & $0.0061(15)$ & $0.0010(16)$ \\
C1 & $0.039(2)$ & $0.041(2)$ & $0.044(2)$ & $0.0106(17)$ & $0.0078(17)$ & $0.0082(18)$ \\
C2 & $0.057(3)$ & $0.042(2)$ & $0.053(3)$ & $0.007(2)$ & $0.015(2)$ & $0.014(2)$ \\
C3 & $0.058(3)$ & $0.041(2)$ & $0.055(3)$ & $0.011(2)$ & $0.018(2)$ & $0.002(2)$ \\
C4 & $0.046(2)$ & $0.056(3)$ & $0.043(2)$ & $0.019(2)$ & $0.0111(18)$ & $0.002(2)$ \\
C5 & $0.079(3)$ & $0.058(3)$ & $0.052(3)$ & $0.013(2)$ & $0.030(2)$ & $0.017(2)$ \\
C6 & $0.077(3)$ & $0.037(2)$ & $0.056(3)$ & $0.006(2)$ & $0.025(2)$ & $0.010(2)$ \\
C7 & $0.038(2)$ & $0.046(2)$ & $0.039(2)$ & $0.0147(18)$ & $0.0014(17)$ & $0.0022(18)$ \\
C8 & $0.044(2)$ & $0.060(3)$ & $0.057(3)$ & $0.020(2)$ & $0.011(2)$ & $0.014(2)$ \\
C9 & $0.056(3)$ & $0.080(3)$ & $0.052(3)$ & $0.027(2)$ & $0.021(2)$ & $0.013(2)$ \\
C12 & $0.0968(10)$ & $0.0802(10)$ & $0.0634(8)$ & $0.0332(8)$ & $0.0091(7)$ & $0.0040(7)$ \\
S3 & $0.0483(6)$ & $0.0431(6)$ & $0.0643(7)$ & $0.0136(5)$ & $0.0182(5)$ & $0.0104(5)$ \\
S4 & $0.0632(7)$ & $0.0641(8)$ & $0.0448(6)$ & $0.0112(6)$ & $0.0121(5)$ & $-0.0066(5)$ \\
O4 & $0.0518(18)$ & $0.073(2)$ & $0.084(2)$ & $0.0203(16)$ & $0.0285(17)$ & $0.0204(19)$ \\
O5 & $0.084(2)$ & $0.0379(17)$ & $0.087(3)$ & $0.0158(16)$ & $0.0302(19)$ & $0.0047(16)$ \\
O6 & $0.097(3)$ & $0.066(2)$ & $0.071(2)$ & $0.021(2)$ & $0.028(2)$ & $0.0261(19)$ \\
N4 & $0.052(2)$ & $0.041(2)$ & $0.050(2)$ & $0.0078(17)$ & $0.0055(16)$ & $-0.0003(16)$ \\
& & & & & &
\end{tabular}




\begin{tabular}{lllllll}
$\mathrm{N} 5$ & $0.052(2)$ & $0.044(2)$ & $0.042(2)$ & $0.0137(16)$ & $0.0069(16)$ & $0.0037(16)$ \\
N6 & $0.057(2)$ & $0.051(2)$ & $0.0315(18)$ & $0.0168(17)$ & $0.0033(16)$ & $0.0056(16)$ \\
C10 & $0.038(2)$ & $0.039(2)$ & $0.058(3)$ & $0.0082(18)$ & $0.0031(18)$ & $0.0137(19)$ \\
C11 & $0.056(3)$ & $0.050(3)$ & $0.061(3)$ & $-0.003(2)$ & $0.008(2)$ & $0.020(2)$ \\
C12 & $0.059(3)$ & $0.039(3)$ & $0.068(3)$ & $-0.001(2)$ & $-0.006(2)$ & $0.013(2)$ \\
C13 & $0.057(3)$ & $0.047(3)$ & $0.058(3)$ & $0.021(2)$ & $0.001(2)$ & $0.014(2)$ \\
C14 & $0.057(3)$ & $0.059(3)$ & $0.063(3)$ & $0.007(2)$ & $0.017(2)$ & $0.015(2)$ \\
C15 & $0.043(2)$ & $0.047(3)$ & $0.072(3)$ & $0.0000(19)$ & $0.016(2)$ & $0.006(2)$ \\
C16 & $0.038(2)$ & $0.050(3)$ & $0.040(2)$ & $0.0146(18)$ & $0.0011(17)$ & $0.0015(19)$ \\
C17 & $0.052(3)$ & $0.072(3)$ & $0.047(3)$ & $0.016(2)$ & $0.010(2)$ & $0.011(2)$ \\
C18 & $0.072(3)$ & $0.073(3)$ & $0.049(3)$ & $0.016(3)$ & $0.018(2)$ & $0.011(2)$ \\
O7 & $0.077(3)$ & $0.065(2)$ & $0.093(3)$ & $0.030(2)$ & $0.013(2)$ & $0.0167(19)$ \\
O8 & $0.056(2)$ & $0.146(5)$ & $0.131(4)$ & $0.030(3)$ & $0.029(3)$ & $0.013(3)$ \\
\hline
\end{tabular}

Geometric parameters $\left(\AA,{ }^{\circ}\right)$

\begin{tabular}{|c|c|c|c|}
\hline $\mathrm{C} 11-\mathrm{C} 4$ & $1.744(4)$ & $\mathrm{S} 3-\mathrm{O} 5$ & $1.441(3)$ \\
\hline $\mathrm{S} 1-\mathrm{O} 1$ & $1.422(3)$ & $\mathrm{S} 3-\mathrm{N} 4$ & $1.649(4)$ \\
\hline $\mathrm{S} 1-\mathrm{O} 2$ & $1.438(3)$ & $\mathrm{S} 3-\mathrm{C} 10$ & $1.759(5)$ \\
\hline $\mathrm{S} 1-\mathrm{N} 1$ & $1.644(4)$ & $\mathrm{S} 4-\mathrm{C} 16$ & $1.746(4)$ \\
\hline $\mathrm{S} 1-\mathrm{C} 1$ & $1.770(4)$ & $\mathrm{S} 4-\mathrm{C} 18$ & $1.812(5)$ \\
\hline $\mathrm{S} 2-\mathrm{C} 7$ & $1.741(4)$ & $\mathrm{O} 6-\mathrm{C} 17$ & $1.218(6)$ \\
\hline $\mathrm{S} 2-\mathrm{C} 9$ & $1.808(5)$ & $\mathrm{N} 4-\mathrm{N} 5$ & $1.442(5)$ \\
\hline $\mathrm{O} 3-\mathrm{C} 8$ & $1.202(5)$ & $\mathrm{N} 4-\mathrm{H} 4 \mathrm{~N}$ & $0.850(19)$ \\
\hline $\mathrm{N} 1-\mathrm{N} 2$ & $1.440(5)$ & $\mathrm{N} 5-\mathrm{C} 16$ & $1.276(5)$ \\
\hline $\mathrm{N} 1-\mathrm{H} 1 \mathrm{~N}$ & $0.842(19)$ & N6-C17 & $1.353(6)$ \\
\hline $\mathrm{N} 2-\mathrm{C} 7$ & $1.285(5)$ & $\mathrm{N} 6-\mathrm{C} 16$ & $1.381(5)$ \\
\hline $\mathrm{N} 3-\mathrm{C} 7$ & $1.370(5)$ & $\mathrm{N} 6-\mathrm{H} 6 \mathrm{~N}$ & $0.851(19)$ \\
\hline $\mathrm{N} 3-\mathrm{C} 8$ & $1.374(5)$ & $\mathrm{C} 10-\mathrm{C} 11$ & $1.389(6)$ \\
\hline $\mathrm{N} 3-\mathrm{H} 3 \mathrm{~N}$ & 0.851 & $\mathrm{C} 10-\mathrm{C} 15$ & $1.390(6)$ \\
\hline $\mathrm{C} 1-\mathrm{C} 6$ & $1.377(6)$ & $\mathrm{C} 11-\mathrm{C} 12$ & $1.369(7)$ \\
\hline $\mathrm{C} 1-\mathrm{C} 2$ & $1.385(6)$ & $\mathrm{C} 11-\mathrm{H} 11$ & 0.9300 \\
\hline $\mathrm{C} 2-\mathrm{C} 3$ & $1.389(6)$ & $\mathrm{C} 12-\mathrm{C} 13$ & $1.368(6)$ \\
\hline $\mathrm{C} 2-\mathrm{H} 2$ & 0.9300 & $\mathrm{C} 12-\mathrm{H} 12$ & 0.9300 \\
\hline $\mathrm{C} 3-\mathrm{C} 4$ & $1.366(6)$ & $\mathrm{C} 13-\mathrm{C} 14$ & $1.380(6)$ \\
\hline $\mathrm{C} 3-\mathrm{H} 3$ & 0.9300 & $\mathrm{C} 14-\mathrm{C} 15$ & $1.368(6)$ \\
\hline $\mathrm{C} 4-\mathrm{C} 5$ & $1.379(6)$ & $\mathrm{C} 14-\mathrm{H} 14$ & 0.9300 \\
\hline $\mathrm{C} 5-\mathrm{C} 6$ & $1.381(6)$ & $\mathrm{C} 15-\mathrm{H} 15$ & 0.9300 \\
\hline $\mathrm{C} 5-\mathrm{H} 5$ & 0.9300 & $\mathrm{C} 17-\mathrm{C} 18$ & $1.498(7)$ \\
\hline C6- 6 6 & 0.9300 & $\mathrm{C} 18-\mathrm{H} 18 \mathrm{~A}$ & 0.9700 \\
\hline $\mathrm{C} 8-\mathrm{C} 9$ & $1.503(6)$ & $\mathrm{C} 18-\mathrm{H} 18 \mathrm{~B}$ & 0.9700 \\
\hline C9-H9A & 0.9700 & $\mathrm{O} 7-\mathrm{H} 71$ & $0.82(2)$ \\
\hline C9-H9B & 0.9700 & $\mathrm{O} 7-\mathrm{H} 72$ & $0.82(2)$ \\
\hline $\mathrm{Cl} 2-\mathrm{C} 13$ & $1.737(5)$ & $\mathrm{O} 8-\mathrm{H} 81$ & $0.82(2)$ \\
\hline $\mathrm{S} 3-\mathrm{O} 4$ & $1.426(3)$ & $\mathrm{O} 8-\mathrm{H} 82$ & $0.83(2)$ \\
\hline $\mathrm{O} 1-\mathrm{S} 1-\mathrm{O} 2$ & $120.4(2)$ & $\mathrm{O} 4-\mathrm{S} 3-\mathrm{N} 4$ & $107.3(2)$ \\
\hline $\mathrm{O} 1-\mathrm{S} 1-\mathrm{N} 1$ & $105.18(19)$ & $\mathrm{O} 5-\mathrm{S} 3-\mathrm{N} 4$ & $103.2(2)$ \\
\hline
\end{tabular}




\begin{tabular}{|c|c|c|c|}
\hline $\mathrm{O} 2-\mathrm{S} 1-\mathrm{N} 1$ & $105.77(19)$ & $\mathrm{O} 4-\mathrm{S} 3-\mathrm{C} 10$ & $107.8(2)$ \\
\hline $\mathrm{O} 1-\mathrm{S} 1-\mathrm{C} 1$ & $108.45(19)$ & $\mathrm{O} 5-\mathrm{S} 3-\mathrm{C} 10$ & $108.6(2)$ \\
\hline $\mathrm{O} 2-\mathrm{S} 1-\mathrm{C} 1$ & $107.88(19)$ & $\mathrm{N} 4-\mathrm{S} 3-\mathrm{C} 10$ & $109.61(18)$ \\
\hline $\mathrm{N} 1-\mathrm{S} 1-\mathrm{C} 1$ & $108.64(18)$ & $\mathrm{C} 16-\mathrm{S} 4-\mathrm{C} 18$ & $91.4(2)$ \\
\hline $\mathrm{C} 7-\mathrm{S} 2-\mathrm{C} 9$ & $91.4(2)$ & $\mathrm{N} 5-\mathrm{N} 4-\mathrm{S} 3$ & $112.3(3)$ \\
\hline $\mathrm{N} 2-\mathrm{N} 1-\mathrm{S} 1$ & $113.6(3)$ & $\mathrm{N} 5-\mathrm{N} 4-\mathrm{H} 4 \mathrm{~N}$ & $106(3)$ \\
\hline $\mathrm{N} 2-\mathrm{N} 1-\mathrm{H} 1 \mathrm{~N}$ & $106(3)$ & $\mathrm{S} 3-\mathrm{N} 4-\mathrm{H} 4 \mathrm{~N}$ & $97(3)$ \\
\hline $\mathrm{S} 1-\mathrm{N} 1-\mathrm{H} 1 \mathrm{~N}$ & $107(3)$ & $\mathrm{C} 16-\mathrm{N} 5-\mathrm{N} 4$ & $112.6(3)$ \\
\hline $\mathrm{C} 7-\mathrm{N} 2-\mathrm{N} 1$ & $111.2(3)$ & $\mathrm{C} 17-\mathrm{N} 6-\mathrm{C} 16$ & $117.8(4)$ \\
\hline $\mathrm{C} 7-\mathrm{N} 3-\mathrm{C} 8$ & $117.6(4)$ & $\mathrm{C} 17-\mathrm{N} 6-\mathrm{H} 6 \mathrm{~N}$ & $124(3)$ \\
\hline $\mathrm{C} 7-\mathrm{N} 3-\mathrm{H} 3 \mathrm{~N}$ & $122(3)$ & $\mathrm{C} 16-\mathrm{N} 6-\mathrm{H} 6 \mathrm{~N}$ & $117(3)$ \\
\hline $\mathrm{C} 8-\mathrm{N} 3-\mathrm{H} 3 \mathrm{~N}$ & $119(3)$ & $\mathrm{C} 11-\mathrm{C} 10-\mathrm{C} 15$ & $119.4(4)$ \\
\hline $\mathrm{C} 6-\mathrm{C} 1-\mathrm{C} 2$ & $120.4(4)$ & $\mathrm{C} 11-\mathrm{C} 10-\mathrm{S} 3$ & $120.0(3)$ \\
\hline $\mathrm{C} 6-\mathrm{C} 1-\mathrm{S} 1$ & $119.8(3)$ & $\mathrm{C} 15-\mathrm{C} 10-\mathrm{S} 3$ & $120.6(3)$ \\
\hline $\mathrm{C} 2-\mathrm{C} 1-\mathrm{S} 1$ & $119.8(3)$ & $\mathrm{C} 12-\mathrm{C} 11-\mathrm{C} 10$ & $120.1(4)$ \\
\hline $\mathrm{C} 1-\mathrm{C} 2-\mathrm{C} 3$ & $119.6(4)$ & $\mathrm{C} 12-\mathrm{C} 11-\mathrm{H} 11$ & 119.9 \\
\hline $\mathrm{C} 1-\mathrm{C} 2-\mathrm{H} 2$ & 120.2 & $\mathrm{C} 10-\mathrm{C} 11-\mathrm{H} 11$ & 119.9 \\
\hline $\mathrm{C} 3-\mathrm{C} 2-\mathrm{H} 2$ & 120.2 & $\mathrm{C} 13-\mathrm{C} 12-\mathrm{C} 11$ & $119.7(4)$ \\
\hline $\mathrm{C} 4-\mathrm{C} 3-\mathrm{C} 2$ & $119.0(4)$ & $\mathrm{C} 13-\mathrm{C} 12-\mathrm{H} 12$ & 120.1 \\
\hline $\mathrm{C} 4-\mathrm{C} 3-\mathrm{H} 3$ & 120.5 & $\mathrm{C} 11-\mathrm{C} 12-\mathrm{H} 12$ & 120.1 \\
\hline $\mathrm{C} 2-\mathrm{C} 3-\mathrm{H} 3$ & 120.5 & $\mathrm{C} 12-\mathrm{C} 13-\mathrm{C} 14$ & $121.2(5)$ \\
\hline $\mathrm{C} 3-\mathrm{C} 4-\mathrm{C} 5$ & $122.1(4)$ & $\mathrm{C} 12-\mathrm{C} 13-\mathrm{Cl} 2$ & $119.4(4)$ \\
\hline $\mathrm{C} 3-\mathrm{C} 4-\mathrm{Cl} 1$ & $118.6(3)$ & $\mathrm{C} 14-\mathrm{C} 13-\mathrm{Cl} 2$ & $119.4(4)$ \\
\hline $\mathrm{C} 5-\mathrm{C} 4-\mathrm{Cl1}$ & $119.2(3)$ & $\mathrm{C} 15-\mathrm{C} 14-\mathrm{C} 13$ & $119.4(4)$ \\
\hline $\mathrm{C} 4-\mathrm{C} 5-\mathrm{C} 6$ & $118.7(4)$ & $\mathrm{C} 15-\mathrm{C} 14-\mathrm{H} 14$ & 120.3 \\
\hline $\mathrm{C} 4-\mathrm{C} 5-\mathrm{H} 5$ & 120.7 & $\mathrm{C} 13-\mathrm{C} 14-\mathrm{H} 14$ & 120.3 \\
\hline $\mathrm{C} 6-\mathrm{C} 5-\mathrm{H} 5$ & 120.7 & $\mathrm{C} 14-\mathrm{C} 15-\mathrm{C} 10$ & $120.3(4)$ \\
\hline $\mathrm{C} 1-\mathrm{C} 6-\mathrm{C} 5$ & $120.2(4)$ & $\mathrm{C} 14-\mathrm{C} 15-\mathrm{H} 15$ & 119.9 \\
\hline $\mathrm{C} 1-\mathrm{C} 6-\mathrm{H} 6$ & 119.9 & $\mathrm{C} 10-\mathrm{C} 15-\mathrm{H} 15$ & 119.9 \\
\hline $\mathrm{C} 5-\mathrm{C} 6-\mathrm{H} 6$ & 119.9 & $\mathrm{~N} 5-\mathrm{C} 16-\mathrm{N} 6$ & $118.7(4)$ \\
\hline $\mathrm{N} 2-\mathrm{C} 7-\mathrm{N} 3$ & $119.3(4)$ & $\mathrm{N} 5-\mathrm{C} 16-\mathrm{S} 4$ & $129.9(3)$ \\
\hline $\mathrm{N} 2-\mathrm{C} 7-\mathrm{S} 2$ & $128.6(3)$ & $\mathrm{N} 6-\mathrm{C} 16-\mathrm{S} 4$ & $111.3(3)$ \\
\hline $\mathrm{N} 3-\mathrm{C} 7-\mathrm{S} 2$ & $112.1(3)$ & $\mathrm{O} 6-\mathrm{C} 17-\mathrm{N} 6$ & $124.2(4)$ \\
\hline $\mathrm{O} 3-\mathrm{C} 8-\mathrm{N} 3$ & $123.9(4)$ & $\mathrm{O} 6-\mathrm{C} 17-\mathrm{C} 18$ & $124.6(4)$ \\
\hline $\mathrm{O} 3-\mathrm{C} 8-\mathrm{C} 9$ & $125.7(4)$ & $\mathrm{N} 6-\mathrm{C} 17-\mathrm{C} 18$ & $111.2(4)$ \\
\hline $\mathrm{N} 3-\mathrm{C} 8-\mathrm{C} 9$ & $110.4(4)$ & $\mathrm{C} 17-\mathrm{C} 18-\mathrm{S} 4$ & $108.0(3)$ \\
\hline $\mathrm{C} 8-\mathrm{C} 9-\mathrm{S} 2$ & $108.4(3)$ & $\mathrm{C} 17-\mathrm{C} 18-\mathrm{H} 18 \mathrm{~A}$ & 110.1 \\
\hline $\mathrm{C} 8-\mathrm{C} 9-\mathrm{H} 9 \mathrm{~A}$ & 110.0 & $\mathrm{~S} 4-\mathrm{C} 18-\mathrm{H} 18 \mathrm{~A}$ & 110.1 \\
\hline $\mathrm{S} 2-\mathrm{C} 9-\mathrm{H} 9 \mathrm{~A}$ & 110.0 & $\mathrm{C} 17-\mathrm{C} 18-\mathrm{H} 18 \mathrm{~B}$ & 110.1 \\
\hline $\mathrm{C} 8-\mathrm{C} 9-\mathrm{H} 9 \mathrm{~B}$ & 110.0 & $\mathrm{~S} 4-\mathrm{C} 18-\mathrm{H} 18 \mathrm{~B}$ & 110.1 \\
\hline $\mathrm{S} 2-\mathrm{C} 9-\mathrm{H} 9 \mathrm{~B}$ & 110.0 & $\mathrm{H} 18 \mathrm{~A}-\mathrm{C} 18-\mathrm{H} 18 \mathrm{~B}$ & 108.4 \\
\hline $\mathrm{H} 9 \mathrm{~A}-\mathrm{C} 9-\mathrm{H} 9 \mathrm{~B}$ & 108.4 & $\mathrm{H} 71-\mathrm{O} 7-\mathrm{H} 72$ & $98(6)$ \\
\hline $\mathrm{O} 4-\mathrm{S} 3-\mathrm{O} 5$ & $119.9(2)$ & $\mathrm{H} 81-\mathrm{O} 8-\mathrm{H} 82$ & $94(7)$ \\
\hline $\mathrm{O} 1-\mathrm{S} 1-\mathrm{N} 1-\mathrm{N} 2$ & $172.9(3)$ & $\mathrm{O} 4-\mathrm{S} 3-\mathrm{N} 4-\mathrm{N} 5$ & $-59.2(3)$ \\
\hline $\mathrm{O} 2-\mathrm{S} 1-\mathrm{N} 1-\mathrm{N} 2$ & $-58.6(3)$ & $\mathrm{O} 5-\mathrm{S} 3-\mathrm{N} 4-\mathrm{N} 5$ & $173.2(3)$ \\
\hline $\mathrm{C} 1-\mathrm{S} 1-\mathrm{N} 1-\mathrm{N} 2$ & $57.0(3)$ & $\mathrm{C} 10-\mathrm{S} 3-\mathrm{N} 4-\mathrm{N} 5$ & $57.6(3)$ \\
\hline $\mathrm{S} 1-\mathrm{N} 1-\mathrm{N} 2-\mathrm{C} 7$ & $111.8(3)$ & $\mathrm{S} 3-\mathrm{N} 4-\mathrm{N} 5-\mathrm{C} 16$ & $109.7(3)$ \\
\hline
\end{tabular}




$\begin{array}{ll}\mathrm{O} 1-\mathrm{S} 1-\mathrm{C} 1-\mathrm{C} 6 & -22.2(4) \\ \mathrm{O} 2-\mathrm{S} 1-\mathrm{C} 1-\mathrm{C} 6 & -154.2(4) \\ \mathrm{N} 1-\mathrm{S} 1-\mathrm{C} 1-\mathrm{C} 6 & 91.6(4) \\ \mathrm{O} 1-\mathrm{S} 1-\mathrm{C} 1-\mathrm{C} 2 & 158.0(3) \\ \mathrm{O} 2-\mathrm{S} 1-\mathrm{C} 1-\mathrm{C} 2 & 26.0(4) \\ \mathrm{N} 1-\mathrm{S} 1-\mathrm{C} 1-\mathrm{C} 2 & -88.2(4) \\ \mathrm{C} 6-\mathrm{C} 1-\mathrm{C} 2-\mathrm{C} 3 & -1.2(6) \\ \mathrm{S} 1-\mathrm{C} 1-\mathrm{C} 2-\mathrm{C} 3 & 178.6(3) \\ \mathrm{C} 1-\mathrm{C} 2-\mathrm{C} 3-\mathrm{C} 4 & -0.1(6) \\ \mathrm{C} 2-\mathrm{C} 3-\mathrm{C} 4-\mathrm{C} 5 & 1.2(7) \\ \mathrm{C} 2-\mathrm{C} 3-\mathrm{C} 4-\mathrm{C} 11 & -177.7(3) \\ \mathrm{C} 3-\mathrm{C} 4-\mathrm{C} 5-\mathrm{C} 6 & -1.1(7) \\ \mathrm{C} 11-\mathrm{C} 4-\mathrm{C} 5-\mathrm{C} 6 & 177.9(4) \\ \mathrm{C} 2-\mathrm{C} 1-\mathrm{C} 6-\mathrm{C} 5 & 1.4(7) \\ \mathrm{S} 1-\mathrm{C} 1-\mathrm{C} 6-\mathrm{C} 5 & -178.4(4) \\ \mathrm{C} 4-\mathrm{C} 5-\mathrm{C} 6-\mathrm{C} 1 & -0.3(7) \\ \mathrm{N} 1-\mathrm{N} 2-\mathrm{C} 7-\mathrm{N} 3 & -176.1(3) \\ \mathrm{N} 1-\mathrm{N} 2-\mathrm{C} 7-\mathrm{S} 2 & 5.0(5) \\ \mathrm{C} 8-\mathrm{N} 3-\mathrm{C} 7-\mathrm{N} 2 & 178.3(4) \\ \mathrm{C} 8-\mathrm{N} 3-\mathrm{C} 7-\mathrm{S} 2 & -2.6(5) \\ \mathrm{C} 9-\mathrm{S} 2-\mathrm{C} 7-\mathrm{N} 2 & -179.0(4) \\ \mathrm{C} 9-\mathrm{S} 2-\mathrm{C} 7-\mathrm{N} 3 & 2.0(3) \\ \mathrm{C} 7-\mathrm{N} 3-\mathrm{C} 8-\mathrm{O} 3 & -178.6(4) \\ \mathrm{C} 7-\mathrm{N} 3-\mathrm{C} 8-\mathrm{C} 9 & 1.6(5) \\ \mathrm{O} 3-\mathrm{C} 8-\mathrm{C} 9-\mathrm{S} 2 & -179.7(4) \\ \mathrm{N} 3-\mathrm{C} 8-\mathrm{C} 9-\mathrm{S} 2 & 0.0(5) \\ \mathrm{C} 7-\mathrm{S} 2-\mathrm{C} 9-\mathrm{C} 8 & -1.1(3) \\ & \end{array}$

$\begin{array}{ll}\mathrm{O} 4-\mathrm{S} 3-\mathrm{C} 10-\mathrm{C} 11 & 23.0(4) \\ \mathrm{O} 5-\mathrm{S} 3-\mathrm{C} 10-\mathrm{C} 11 & 154.4(3) \\ \mathrm{N} 4-\mathrm{S} 3-\mathrm{C} 10-\mathrm{C} 11 & -93.5(4) \\ \mathrm{O} 4-\mathrm{S} 3-\mathrm{C} 10-\mathrm{C} 15 & -157.6(3) \\ \mathrm{O} 5-\mathrm{S} 3-\mathrm{C} 10-\mathrm{C} 15 & -26.3(4) \\ \mathrm{N} 4-\mathrm{S} 3-\mathrm{C} 10-\mathrm{C} 15 & 85.8(4) \\ \mathrm{C} 15-\mathrm{C} 10-\mathrm{C} 11-\mathrm{C} 12 & -0.4(7) \\ \mathrm{S} 3-\mathrm{C} 10-\mathrm{C} 11-\mathrm{C} 12 & 179.0(4) \\ \mathrm{C} 10-\mathrm{C} 11-\mathrm{C} 12-\mathrm{C} 13 & 0.6(7) \\ \mathrm{C} 11-\mathrm{C} 12-\mathrm{C} 13-\mathrm{C} 14 & 0.2(7) \\ \mathrm{C} 11-\mathrm{C} 12-\mathrm{C} 13-\mathrm{C} 12 & -179.1(3) \\ \mathrm{C} 12-\mathrm{C} 13-\mathrm{C} 14-\mathrm{C} 15 & -1.2(7) \\ \mathrm{C} 12-\mathrm{C} 13-\mathrm{C} 14-\mathrm{C} 15 & 178.1(4) \\ \mathrm{C} 13-\mathrm{C} 14-\mathrm{C} 15-\mathrm{C} 10 & 1.3(7) \\ \mathrm{C} 11-\mathrm{C} 10-\mathrm{C} 15-\mathrm{C} 14 & -0.6(7) \\ \mathrm{S} 3-\mathrm{C} 10-\mathrm{C} 15-\mathrm{C} 14 & -179.9(4) \\ \mathrm{N} 4-\mathrm{N} 5-\mathrm{C} 16-\mathrm{N} 6 & -179.6(3) \\ \mathrm{N} 4-\mathrm{N} 5-\mathrm{C} 16-\mathrm{S} 4 & 0.8(5) \\ \mathrm{C} 17-\mathrm{N} 6-\mathrm{C} 16-\mathrm{N} 5 & -174.4(4) \\ \mathrm{C} 17-\mathrm{N} 6-\mathrm{C} 16-\mathrm{S} 4 & 5.3(5) \\ \mathrm{C} 18-\mathrm{S} 4-\mathrm{C} 16-\mathrm{N} 5 & 174.5(4) \\ \mathrm{C} 18-\mathrm{S} 4-\mathrm{C} 16-\mathrm{N} 6 & -5.1(3) \\ \mathrm{C} 16-\mathrm{N} 6-\mathrm{C} 17-\mathrm{O} 6 & 177.6(4) \\ \mathrm{C} 16-\mathrm{N} 6-\mathrm{C} 17-\mathrm{C} 18 & -2.1(5) \\ \mathrm{O} 6-\mathrm{C} 17-\mathrm{C} 18-\mathrm{S} 4 & 178.5(4) \\ \mathrm{N} 6-\mathrm{C} 17-\mathrm{C} 18-\mathrm{S} 4 & -1.8(5) \\ \mathrm{C} 16-\mathrm{S} 4-\mathrm{C} 18-\mathrm{C} 17 & 3.9(3) \\ & \end{array}$

Hydrogen-bond geometry $\left(A,{ }^{\circ}\right)$

\begin{tabular}{lllll}
\hline$D-\mathrm{H} \cdots A$ & $D-\mathrm{H}$ & $\mathrm{H} \cdots A$ & $D \cdots A$ & $D-\mathrm{H} \cdots A$ \\
\hline $\mathrm{N} 1-\mathrm{H} 1 N \cdots \mathrm{O} 7^{\mathrm{i}}$ & $0.84(2)$ & $2.07(2)$ & $2.900(6)$ & $168(4)$ \\
$\mathrm{N} 3-\mathrm{H} 3 N \cdots \mathrm{N} 5^{\mathrm{ii}}$ & $0.85(2)$ & $2.07(2)$ & $2.895(5)$ & $162(4)$ \\
$\mathrm{C} 9-\mathrm{H} 9 B \cdots \mathrm{O} 2^{\mathrm{ii}}$ & 0.97 & 2.42 & $3.236(6)$ & 141 \\
$\mathrm{~N} 4-\mathrm{H} 4 N \cdots \mathrm{O} 8$ & $0.85(2)$ & $1.95(2)$ & $2.788(6)$ & $168(4)$ \\
$\mathrm{N} 6-\mathrm{H} 6 N \cdots \mathrm{N} 2^{\mathrm{iii}}$ & $0.85(2)$ & $1.97(2)$ & $2.808(5)$ & $170(4)$ \\
$\mathrm{C} 15-\mathrm{H} 15 \cdots \mathrm{O} 1^{\text {iv }}$ & 0.93 & 2.55 & $3.355(5)$ & 145 \\
$\mathrm{O} 7-\mathrm{H} 71 \cdots \mathrm{O} 5$ & $0.82(2)$ & $2.08(3)$ & $2.868(5)$ & $162(6)$ \\
$\mathrm{O} 7-\mathrm{H} 72 \cdots \mathrm{O} 6^{\text {iv }}$ & $0.82(2)$ & $1.99(2)$ & $2.810(5)$ & $174(6)$ \\
$\mathrm{O} 8-\mathrm{H} 81 \cdots \mathrm{O} 4^{\mathrm{ii}}$ & $0.82(2)$ & $2.35(6)$ & $2.987(6)$ & $136(7)$ \\
$\mathrm{O} 8-\mathrm{H} 81 \cdots \mathrm{O} 7^{\mathrm{ii}}$ & $0.82(2)$ & $2.50(7)$ & $3.034(7)$ & $124(7)$ \\
$\mathrm{O} 8-\mathrm{H} 82 \cdots \mathrm{O} 3^{\mathrm{iii}}$ & $0.83(2)$ & $2.37(3)$ & $3.159(7)$ & $159(8)$
\end{tabular}

Symmetry codes: (i) $x-1, y+1, z$; (ii) $x-1, y, z$; (iii) $x+1, y, z$; (iv) $x, y-1, z$. 